\title{
Expression and clinical significance of serum amyloid $A$ and interleukin-6 in patients with acute exacerbation of chronic obstructive pulmonary disease
}

\author{
YONGTAO WEI $^{1}$, SONGXIA WANG ${ }^{2}$, DONGPING WANG ${ }^{1}$ and CHENG LIU ${ }^{1}$ \\ ${ }^{1}$ Clinical Laboratory and ${ }^{2}$ Blood Transfusion Department, Qingdao Eighth \\ People's Hospital, Qingdao, Shandong 266100, P.R. China
}

Received September 18, 2019; Accepted November 14, 2019

DOI: $10.3892 /$ etm.2019.8366

\begin{abstract}
Expression and clinical significance of serum amyloid A (SAA) and interleukin-6 (IL-6) in patients with acute exacerbation of chronic obstructive pulmonary disease (AECOPD) were investigated. Fifty-one patients with AECOPD (acute group, AG) and 51 patients with stable chronic obstructive pulmonary disease (stable group, SG) admitted to Qingdao Eighth People's Hospital were selected. The expression levels of SAA and IL-6 in peripheral blood of patients in the two groups were detected and compared before and after treatment. Pearson analysis was used in the correlation between SAA and IL-6 and Receiver operating characteristic (ROC) curve was employd to analyze the predictive value of SAA and IL-6 for AECOPD progression. Logistic regression analysis was used to analyze the risk factors of AECOPD patients. The expression levels of SAA and IL- 6 of patients in AG were significantly higher than those in $\mathrm{SG}(\mathrm{P}<0.05)$. Pearson analysis showed that SAA was positively correlated with IL-6 expression $(\mathrm{P}<0.05)$. ROC curve analysis showed that AUC predicted by SAA and IL-6 for AECOPD progress was 0.789 and $0.762(\mathrm{P}<0.05)$. Logistic regression analysis showed that SAA and IL-6 were prediction indexes of AECOPD progression. The levels of SAA and IL-6 were significantly increased during AECOPD and effectively predicted the progress of AECOPD and is a risk factor affecting AECOPD patients.
\end{abstract}

\section{Introduction}

Chronic obstructive pulmonary disease (COPD) is one of the most common lung diseases observed in clinical practice

Correspondence to: Dr Songxia Wang, Blood Transfusion Department, Qingdao Eighth People's Hospital, 84 Fengshan Road, Licang, Qingdao, Shandong 266100, P.R. China

E-mail: s3f1vs@163.com

Key words: serum amyloid A, interleukin-6, acute exacerbation of chronic obstructive pulmonary disease, acute stable of chronic obstructive pulmonary disease, risk factor and the third leading cause of death in the world $(1,2)$. COPD prevalence rate of people aged over 40 years is $10.1 \%$ in the world and $8.2 \%$ in China (3). According to relevant data, the death rate of COPD inpatients in the United States was $4.8 \%$ in 2002 and the average hospitalization cost of patients was as high as $\$ 22,187$ (4). COPD mainly manifests as airflow obstruction and lung inflammation (5). Due to deterioration of lung function, people with severe COPD are usually unable to participate in normal physical activities (6). Acute exacerbation of COPD (AECOPD) is associated with acute inflammation and infection and increases morbidity and mortality (7). However, little is known about the prognostic consequences of these deterioration (8).

Serum amyloid A (SAA), a precursor protein of amyloid fibrils (9), is an evolved and highly conserved acute phase protein secreted mainly by hepatocytes (10). Some studies have pointed out that SAA may be a potential marker of lung cancer (11). However, at present, there are still few studies on the role of SAA in AECOPD. Interleukin-6 (IL-6) is one of the most important inflammatory cytokines (12). It was originally described as B-stimulating factor 2, which induces B lymphocytes to produce immunoglobulin (13). It can be rapidly synthesized in the case of infection and tissue damage (14). Relevant data showed that IL-6 plays a key role in the pathogenesis of inflammatory diseases and the homeostasis of nerve tissue (15). COPD is characterized by local and systemic inflammation. Because inflammation plays a key role in the progression, course and severity of COPD, inflammatory markers have the potential to improve current diagnosis and prognosis methods (16). However, few studies have systematically investigated the levels of inflammatory cytokines in patients with AECOPD.

Therefore, we explored the expression of SAA and IL-6 in the peripheral blood serum of AECOPD patients through experiments to provide accurate basis for future clinical diagnosis and treatment of AECOPD.

\section{Patients and methods}

Baseline data of patients. In this study, 51 patients with acute exacerbation of chronic obstructive pulmonary disease (AG) admitted to Qingdao Eighth People's Hospital (Qingdao, China) from March 2010 to February 2015 were selected, 
Table I. Comparison of the baseline data of COPD patients between the two groups [n (\%)].

\begin{tabular}{|c|c|c|c|c|}
\hline Items & AG (51) & SG (51) & $\mathrm{t}$ or $\chi^{2}$ & P-value \\
\hline Sex & & & 0.168 & 0.682 \\
\hline Male & $31(60.78)$ & $33(64.71)$ & & \\
\hline Female & $20(41.18)$ & $18(35.29)$ & & \\
\hline Age/years & $56.31 \pm 6.74$ & $56.62 \pm 6.49$ & 0.237 & 0.813 \\
\hline BMI $\left(\mathrm{kg} / \mathrm{m}^{2}\right)$ & $22.47 \pm 3.83$ & $22.15 \pm 4.62$ & 0.381 & 0.704 \\
\hline Place of residence & & & 0.056 & 0.813 \\
\hline City & 39 (76.47) & 40 (78.43) & & \\
\hline Rural & $12(23.53)$ & $11(21.57)$ & & \\
\hline Ethnicity & & & 0.703 & 0.402 \\
\hline Han & $45(88.24)$ & $42(82.35)$ & & \\
\hline Minority & $6(11.76)$ & $9(17.65)$ & & \\
\hline Smoking history & & & 0.050 & 0.822 \\
\hline Yes & $38(74.51)$ & $37(72.55)$ & & \\
\hline No & $13(25.49)$ & $14(27.45)$ & & \\
\hline Drinking history & & & 0.177 & 0.674 \\
\hline Yes & $33(64.71)$ & $35(68.63)$ & & \\
\hline No & $18(35.29)$ & $16(31.37)$ & & \\
\hline Education level & & & 0.403 & 0.526 \\
\hline$<$ High school & $15(29.41)$ & $18(35.29)$ & & \\
\hline$\geq$ High school & $36(70.59)$ & $33(64.71)$ & & \\
\hline Marital status & & & 0.297 & 0.586 \\
\hline Married & $42(82.35)$ & $44(86.27)$ & & \\
\hline Unmarried & $9(17.65)$ & $7(13.73)$ & & \\
\hline Family history & & & 0.159 & 0.689 \\
\hline Yes & $23(45.10)$ & $21(41.18)$ & & \\
\hline No & $28(54.90)$ & $30(58.82)$ & & \\
\hline
\end{tabular}

COPD, chronic obstructive pulmonary disease; AG, acute group; SG, stable group.

including 31 males and 20 females, aged 45-70 years with an average age of $(56.31 \pm 6.74)$ years. Fifty-one patients with stable chronic obstructive pulmonary disease (SG) were selected, including 33 males and 18 females, aged 44-69 years and an average age of $(56.62 \pm 6.49)$ years. The study was approved by the Ethics Committee of Qingdao Eighth People's Hospital. Signed informed consents were obtained from the patients and/or guardians.

\section{Inclusion and exclusion criteria}

Inclusion criteria. The patients who met the diagnostic criteria of the Spanish management guide of COPD in 2017 (17). The patients received treatment in the above hospital after diagnosis and had complete case data. All the patients agreed to cooperate with the work arrangements of the medical staff in the hospital. Informed consent was signed by the patient or an immediate family member.

Exclusion criteria. Died during treatment; injury of important organs; comorbid with other cardiovascular and cerebrovascular diseases; physical disability; pregnancy; comorbid with other autoimmune diseases; comorbid with other chronic diseases; transferred to another hospital; contraindications of surgery; mental diseases, language dysfunction and diseases affecting the results of this study.

Methods. In both groups, $3 \mathrm{ml}$ of peripheral venous blood was extracted on an empty stomach in the early morning, and the serum was centrifuged for $10 \mathrm{~min}$ at $300 \mathrm{x} \mathrm{g}$ and $28^{\circ} \mathrm{C}$, and then separated and stored at $-80^{\circ} \mathrm{C}$ for measurement. The serum IL-6 and SAA levels were detected by enzyme-linked immunosorbent assay (ELISA). IL-6 detection kit was purchased from Shanghai Chunshi Biotechnology Co., Ltd., item number (CS-13629E). The SAA detection kit was purchased from Shanghai YuanMu Biological Technology Co. Ltd., item number (YM-SX0898). The operation steps were carried out strictly in accordance with the requirements of the manual.

Statistical methods. The experimental results were calculated using SPSS24.0 statistical (Beijing Sitron Weida Information Technology Co., Ltd.). The image was drawn by GraphPad Prism 7. Counting data was expressed as a percentage (\%). The chi-square test was used to compare between the two groups. The measurement data was expressed as mean \pm standard 


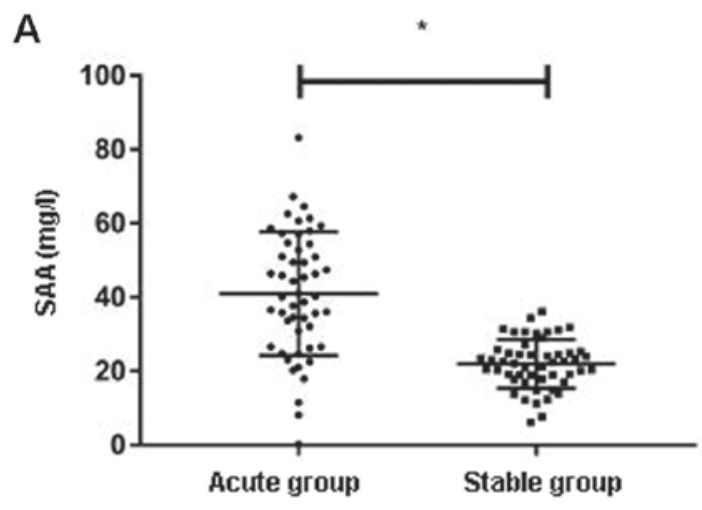

B

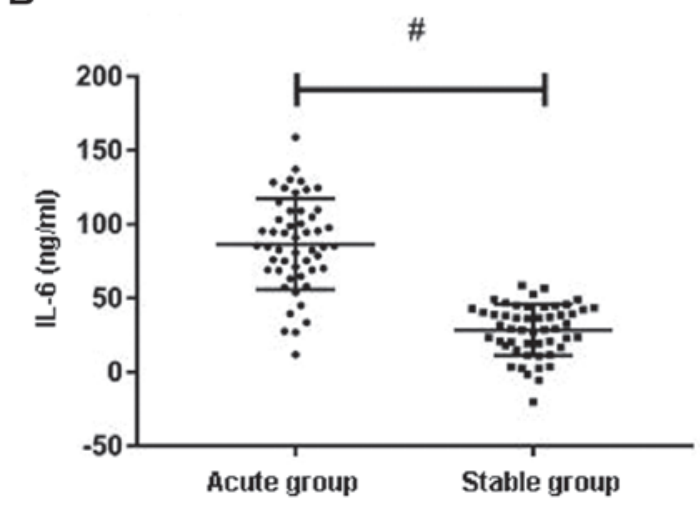

Figure 1. Comparison of SAA and IL-6 between the two groups. (A) The expression levels of SAA in the AG were significantly higher than that in the $\mathrm{SG},{ }^{*} \mathrm{P}<0.05$. (B) The expression levels of IL-6 in the AG were significantly lower than that in the $\mathrm{SG},{ }^{\#} \mathrm{P}<0.05$. SAA, serum amyloid A; IL-6, interleukin-6; AG, acute group; SG, stable group.

deviation (SD). t-test was used for comparison between groups. The receiver operating characteristic (ROC) curve was used to evaluate the diagnostic efficacy and calculate the sensitivity, and specificity. Pearson test was used to analyze the correlation between SAA and IL-6 in serum. Chi-square $\left(\chi^{2}\right)$ test was used for counting data to conduct single factor analysis of AECOPD. The indexes of $\mathrm{P}<0.05$ in single factor analysis were incorporated into logistic multiple regression model (stepwise regression) for multivariate analysis. Independent risk factors related to AECOPD were screened. The difference was statistically significant at $\mathrm{P}<0.05$.

\section{Results}

Comparison of the baseline data of patients between the two groups. The two groups of patients were comparable in baseline data of sex, age and BMI, with no significant difference $(\mathrm{P}>0.05)$ (Table I).

Expression levels of SAA and IL-6 in peripheral blood of patients in the two groups. The results showed that the relative level of SAA expression in serum of AECOPD patients $(40.68 \pm 14.49) \mathrm{ng} / \mathrm{ml}$ was significantly higher than that of patients with stable COPD $(21.58 \pm 6.91) \mathrm{ng} / \mathrm{ml}$, $\mathrm{P}<0.001$. More details are shown in Fig. 1A. The relative level of IL- 6 expression in serum of AECOPD patients $(80.93 \pm 33.52) \mathrm{ng} / \mathrm{ml}$ was significantly higher than that of
Table II. Correlation analysis of SAA and IL-6.

\begin{tabular}{ll}
\hline Items & \multicolumn{1}{c}{ Value } \\
\hline $\mathrm{r}$ & 0.765 \\
$95 \%$ CI & $0.6202-0.8594$ \\
P-value & $<0.001$ \\
\hline
\end{tabular}

SAA, serum amyloid A; IL-6, interleukin-6.

Table III. Predictive value of SAA and IL-6 for AECOPD progression.

\begin{tabular}{lll}
\hline Items & \multicolumn{1}{c}{ SAA } & \multicolumn{1}{c}{ IL-6 } \\
\hline AUC & 0.789 & 0.762 \\
SE & 0.045 & 0.046 \\
$95 \%$ CI & $0.7002-0.8777$ & $0.6721-0.8527$ \\
P-value & $<0.001$ & $<0.001$ \\
Cut-off & $10.06 \mathrm{ng} / \mathrm{ml}$ & $15.69 \mathrm{ng} / \mathrm{ml}$ \\
Sensitivity (\%) & 82.35 & 88.24 \\
Specificity (\%) & 68.63 & 49.02 \\
\hline
\end{tabular}

SAA, serum amyloid A; IL-6, interleukin-6; SE, standard error; AECOPD, acute exacerbation of chronic obstructive pulmonary disease.

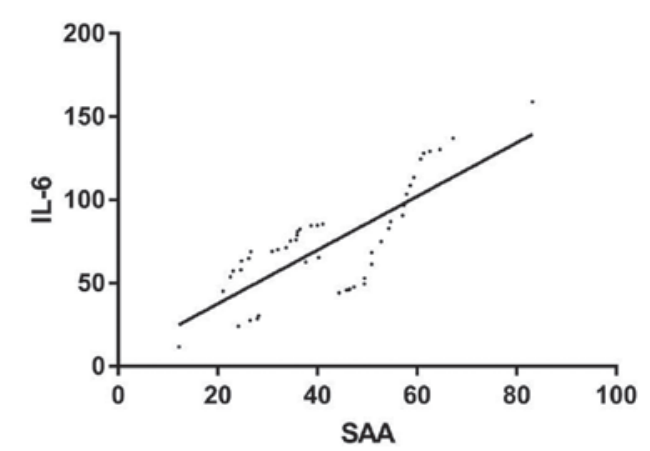

Figure 2. Pearson correlation analysis shows that SAA level in serum of patients in AG was positively correlated with IL-6 ( $\mathrm{r}=0.765, \mathrm{P}<0.001)$. SAA, serum amyloid A; IL-6, interleukin-6; AG, acute group.

patients with stable COPD $(27.68 \pm 17.27) \mathrm{ng} / \mathrm{ml}, \mathrm{P}<0.001$ (Fig. 1B).

Correlation analysis of SAA and IL-6 in AG. Pearson correlation analysis showed that SAA level in serum of patients in AG was positively correlated with IL-6 $(r=0.765, \mathrm{P}<0.001)$ (Table II and Fig. 2).

Predictive value of SAA and IL-6 for AECOPD progression. When the cut-off value was $10.06 \mathrm{ng} / \mathrm{ml}$, the sensitivity of SAA to AECOPD progression was $82.35 \%$ and the specificity was $68.63 \%$. When the cut-off value was $15.69 \mathrm{ng} / \mathrm{ml}$, the sensitivity of IL-6 to AECOPD progression was $88.24 \%$ and the specificity was $49.02 \%$ (Table III and Fig. 3A and B). 
Table IV. Assignments.

Index

Assignment

Diabetes mellitus

Respiratory failure

$\mathrm{SAA}(\mathrm{mg} / \mathrm{l})$

IL-6 (ng/1)
$\mathrm{No}=0 ; \mathrm{Yes}=1$

$\mathrm{No}=0 ; \mathrm{Yes}=1$

The data conformed to continuous variables and were analyzed with original data. The data conformed to continuous variables and were analyzed with original data.

SAA, serum amyloid A; IL-6, interleukin-6.

Table V. Multivariate regression analysis.

\begin{tabular}{lccccrr}
\hline Items & $\mathrm{B}$ & $\mathrm{SE}$ & Wald & P-value & OR & 95\% CI \\
\hline Diabetes mellitus & 1.922 & 0.431 & 18.832 & 0.020 & 1.234 & $2.281-19.694$ \\
Respiratory failure & 1.613 & 0.784 & 4.238 & 0.040 & 5.020 & $1.080-23.324$ \\
SAA & 1.172 & 0.536 & 4.744 & 0.028 & 3.227 & $1.124-9.256$ \\
IL-6 & 0.701 & 0.239 & 8.705 & 0.002 & 2.019 & $1.267-3.218$ \\
\hline
\end{tabular}

SE, standard error; SAA, serum amyloid A; IL-6, interleukin-6.

\section{A}

ROC curve: ROC of Data 4

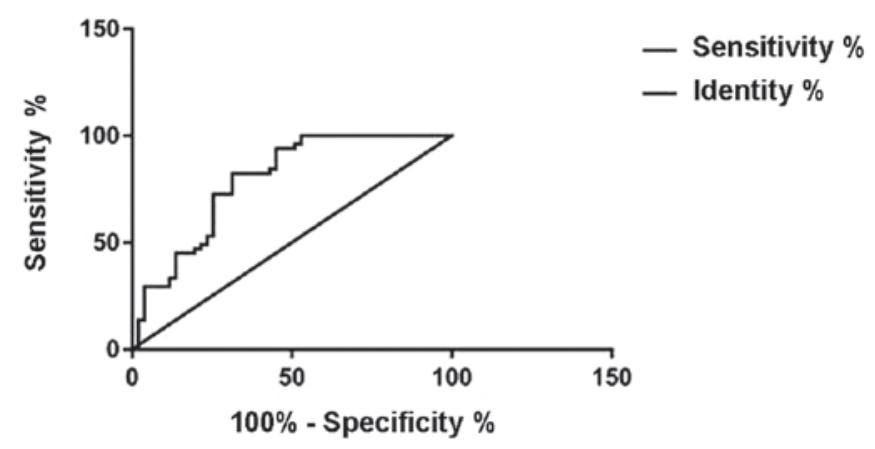

B

ROC curve: ROC of Data 5

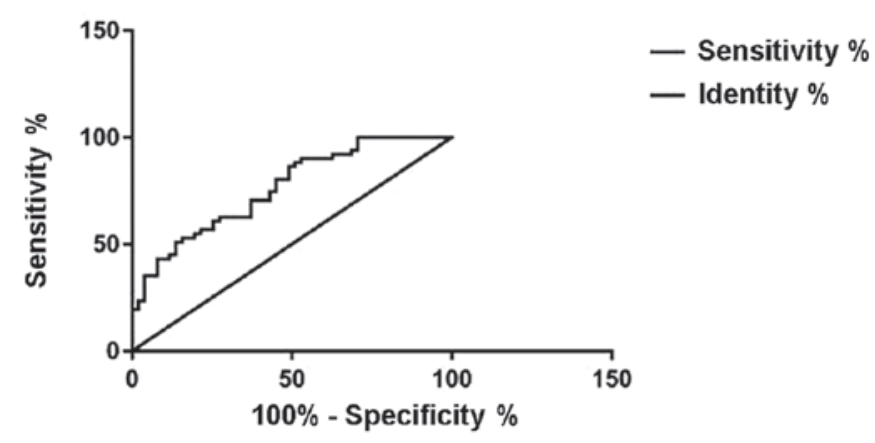

Figure 3. Diagnostic value of SAA and IL-6 for AECOPD. (A) ROC curve of SAA prediction of AECOPD. Cut-off value was 10.06, AUC was 0.789, SE was $0.045,95 \%$ CI was $0.7002-0.8777$, sensitivity was $82.35 \%$ and specificity was $68.63 \%, \mathrm{P}<0.001$. (B) ROC curve of SAA prediction of AECOPD. Cut-off value was 15.69 , AUC was 0.762 , SE was $0.046,95 \%$ CI was $0.678-0.859$, sensitivity was $88.24 \%$ and specificity was $49.02 \%, \mathrm{P}<0.001$. SAA, serum amyloid A; IL-6, interleukin-6; AECOPD, acute exacerbation of chronic obstructive pulmonary disease; ROC, receiver operating characteristic; SE, standard error.
Analysis of single risk factors in the two groups. The indicators with differences in the above results were taken as the results of single factor analysis and assigned values (Table IV). SPSS was used to select LR: forward for multivariate regression analysis. The results showed that diabetes was an independent risk factor for affecting AECOPD (OR, 1.234; 95\% CI, 2.281-19.694). Respiratory failure was an independent risk factor for affecting AECOPD (OR, 5.020; 95\% CI, 1.080-23.324). SAA was an independent risk factor for affecting AECOPD (OR, 3.227; 95\% CI, 1.124-9.256). IL-6 was an independent risk factor for affecting AECOPD (OR, 2.019; 95\% CI, 1.267-3.218) (Table V).

\section{Discussion}

COPD is a common, preventable and treatable disease, which is caused by airway and/or alveolar abnormalities and is usually caused by exposure to harmful particles or gases $(18,19)$. Moreover, frequent hospitalization leads to a significant decrease in the quality of life of patients (20) and severe acute exacerbation also predicts a poor prognosis, with mortality rates exceeding, respectively, 20 and $50 \%$ in 1 and 5 years (21).

SAA is a major acute plasma protein, which can regulate innate immunity and cholesterol homeostasis (22). SAA has a significant relationship with acute phase reaction. Serum level rises up to 1,000 times within $24 \mathrm{~h}$ (23). This is the same as C-reactive protein (CRP). SAA can be used as a diagnostic, prognostic or therapeutic follow-up marker for many diseases $(24,25)$. Cytokines are effective inducers of SAA in hepatocytes (26). Relevant literature shows that the synthesis of SAA is regulated by IL-6 (27). IL-6 is a multi-effect cytokine with known multiple functions in immune regulation, inflammation and tumorigenesis $(28,29)$. COPD is an inflammatory and irreversible lung disease (30). Biological 
medicines for inflammatory cytokine IL-6 are increasingly considered as treatment methods for chronic diseases and cancers (31). However, the role of IL-6 in AECOPD is still less elaborated. Therefore, analyzing the impact of SAA and IL-6 on AECOPD, is not only of great significance for future clinical screening of AECOPD, but also provides new ideas for potential therapeutic targets of AECOPD in the future.

The results of this study showed that the expression levels of SAA and IL-6 were significantly up-regulated in COPD patients, suggesting that SAA and IL-6 may participate in the development and progression of AECOPD, which is consistent with the research results of Li et al (32) and Chen et al (33) and support the results of this study. According to Pearson correlation analysis, SAA level in serum of patients in AG was positively correlated with IL-6 $(r=0.765, \mathrm{P}<0.001)$, which shows serious tissue damage in AECOPD progression. At this time, the content of pro-inflammatory cytokine IL-16 increases significantly and regulates the accelerated secretion of SAA. By drawing ROC curve of SAA and IL-6, we found SAA AUC $=0.789,95 \%$ CI, 0.7002-0.8777, while IL-6 $\mathrm{AUC}=0.762,95 \% \mathrm{CI}, 0.6721-0.8527$. This showed that SAA and IL- 6 have a very good predictive value in the prediction of AECOPD progress. Moreover, the study of Landi et al (34) showed that IL-16 has a good predictive value for myogenic myelitis. Previous studies also confirmed that SAA has a good predictive effect for postoperative infection of osteosarcoma and ovarian cancer $(35,36)$, which also confirms our viewpoint. Through multivariate regression analysis, diabetes, respiratory failure, SAA and IL-6 are independent risk factors for affecting AECOPD, suggesting that in clinical situation of AECOPD patients should be paid attention during treatment in clinic. Patients with longer course and more severe disease should be paid more attention during the treatment process.

The purpose of this study was to explore and analyze the role of SAA and IL-6 in AECOPD. However, the number of cases is limited, and the experimental period is short. Thus, further study is required.

In conclusion, the levels of SAA and IL-6 are significantly increased during AECOPD and they are positively correlated. They may participate in the development and progression of AECOPD and can effectively predict and affect the progress of AECOPD.

\section{Acknowledgements}

Not applicable.

\section{Funding}

No funding was received.

\section{Availability of data and materials}

The datasets used and/or analyzed during the current study are available from the corresponding author on reasonable request.

\section{Authors' contributions}

YW wrote the manuscript. YW and SW conceived and designed the study. YW and DW were responsible for the collection and analysis of the experimental data. SW and CL interpreted the data and drafted the manuscript. DW and CL revised the manuscript critically for important intellectual content. All authors read and approved the final manuscript.

\section{Ethics approval and consent to participate}

The study was approved by the Ethics Committee of Qingdao Eighth People's Hospital (Qingdao, China). Patients who participated in this research had complete clinical data. Signed informed consents were obtained from the patients and/or guardians.

\section{Patient consent for publication}

Not applicable.

\section{Conflict of interest}

The authors declare that they have no competing interests.

\section{References}

1. Santibáñez M, Garrastazu R, Ruiz-Nuñez M, Helguera JM, Arenal S, Bonnardeux C, León C and García-Rivero JL: Predictors of hospitalized exacerbations and mortality in chronic obstructive pulmonary disease. PLoS One 11: e0158727, 2016.

2. Wain LV, Shrine N, Artigas MS, Erzurumluoglu AM, Noyvert B, Bossini-Castillo L, Obeidat M, Henry AP, Portelli MA, Hall RJ, et al; Understanding Society Scientific Group; Geisinger-Regeneron DiscovEHR Collaboration: Genome-wide association analyses for lung function and chronic obstructive pulmonary disease identify new loci and potential druggable targets. Nat Genet 49: 416-425, 2017.

3. Zhou Y, Zhong NS, Li X, Chen S, Zheng J, Zhao D, Yao W, Zhi R, Wei L, He B, et al: Tiotropium in early-stage chronic obstructive pulmonary disease. N Engl J Med 377: 923-935, 2017.

4. Jinjuvadia C, Jinjuvadia R, Mandapakala C, Durairajan N, Liangpunsakul S and Soubani AO: Trends in outcomes, financial burden, and mortality for acute exacerbation of chronic obstructive pulmonary disease (COPD) in the United States from 2002 to 2010. COPD14: 72-79, 2017.

5. Cebron Lipovec N, Beijers RJ, van den Borst B, Doehner W, Lainscak $M$ and Schols AM: The prevalence of metabolic syndrome in chronic obstructive pulmonary disease: A systematic review. COPD 13: 399-406, 2016.

6. US Preventive Services Task Force (USPSTF), Siu AL, Bibbins-Domingo K, Grossman DC, Davidson KW, Epling JW Jr, García FA, Gillman M, Kemper AR, Krist AH, et al: Screening for chronic obstructive pulmonary disease: US Preventive Services Task Force recommendation statement. JAMA 315: 1372-1377, 2016.

7. Tan DBA, Armitage J, Teo TH, Ong NE, Shin H and Moodley YP: Elevated levels of circulating exosome in COPD patients are associated with systemic inflammation. Respir Med 132: 261-264, 2017.

8. Soler-Cataluña JJ, Martínez-García MA, Román Sánchez P, Salcedo E, Navarro $M$ and Ochando R: Severe acute exacerbations and mortality in patients with chronic obstructive pulmonary disease. Thorax 60: 925-931, 2005.

9. Takase H, Tanaka M, Yamamoto A, Watanabe S, Takahashi S, Nadanaka S, Kitagawa H, Yamada T and Mukai T: Structural requirements of glycosaminoglycans for facilitating amyloid fibril formation of human serum amyloid A. Amyloid 23: 67-75, 2016.

10. Siegmund SV, Schlosser M, Schildberg FA, Seki E, De Minicis S, Uchinami H, Kuntzen C, Knolle PA, Strassburg CP and Schwabe RF: Serum amyloid a induces inflammation, proliferation and cell death in activated hepatic stellate cells PLoS One 11: e0150893, 2016. 
11. Sung HJ, Ahn JM, Yoon YH, Rhim TY, Park CS, Park JY, Lee SY, Kim JW and Cho JY: Identification and validation of SAA as a potential lung cancer biomarker and its involvement in metastatic pathogenesis of lung cancer. J Proteome Res 10: 1383-1395, 2011.

12. Schaper F and Rose-John S: Interleukin-6: Biology, signaling and strategies of blockade. Cytokine Growth Factor Rev 26: 475-487, 2015.

13. Zhou YQ, Liu Z, Liu ZH, Chen SP, Li M, Shahveranov A, Ye DW and Tian YK: Interleukin-6: An emerging regulator of pathological pain. J Neuroinflammation 13: 141, 2016.

14. Jordan SC, Choi J, Kim I, Wu G, Toyoda M, Shin B and Vo A: Interleukin-6, a cytokine critical to mediation of inflammation, autoimmunity and allograft rejection: Therapeutic implications of IL-6 receptor blockade. Transplantation 101: 32-44, 2017.

15. Rothaug M, Becker-Pauly C and Rose-John S: The role of interleukin-6 signaling in nervous tissue. Biochim Biophys Acta 1863A: 1218-1227, 2016.

16. Lopez-Campos JL, Calero-Acuña C, Lopez-Ramirez C Abad-Arranz M, Márquez-Martín E, Ortega-Ruiz F and Arellano E: Implications of the inflammatory response for the identification of biomarkers of chronic obstructive pulmonary disease. Biomark Med 10: 109-122, 2016.

17. Miravitlles M, Soler-Cataluña JJ, Calle M, Molina J, Almagro P, Quintano JA, Trigueros JA, Cosío BG, Casanova C, Antonio Riesco J, et al: Spanish Guidelines for Management of Chronic Obstructive Pulmonary Disease (GesEPOC) 2017. Pharmacological treatment of stable phase. Arch Bronconeumol 53: 324-335, 2017.

18. Vogelmeier CF, Criner GJ, Martínez FJ, Anzueto A, Barnes PJ, Bourbeau J, Celli BR, Chen R, Decramer M, Fabbri LM, et al: Global strategy for the diagnosis, management, and prevention of chronic obstructive lung disease 2017 report. GOLD executive summary. Am J Respir Crit Care Med 195: 557-582, 2017.

19. Dalal AA, Christensen L, Liu F and Riedel AA: Direct costs of chronic obstructive pulmonary disease among managed care patients. Int J Chron Obstruct Pulmon Dis 5: 341-349, 2010.

20. Seemungal TA, Donaldson GC, Paul EA, Bestall JC, Jeffries DJ and Wedzicha JA: Effect of exacerbation on quality of life in patients with chronic obstructive pulmonary disease. Am J Respir Crit Care Med 157: 1418-1422, 1998.

21. Dransfield MT, Kunisaki KM, Strand MJ, Anzueto A, Bhatt SP, Bowler RP, Criner GJ, Curtis JL, Hanania NA, Nath H, et al; COPD Gene Investigators: Acute exacerbations and lung function loss in smokers with and without chronic obstructive pulmonary disease. Am J Respir Crit Care Med 195: 324-330, 2017.
22. Frame NM and Gursky O: Structure of serum amyloid A suggests a mechanism for selective lipoprotein binding and functions: SAA as a hub in macromolecular interaction networks. FEBS Lett 590: 866-879, 2016

23. Sack GH Jr: Serum amyloid A - a review. Mol Med 24: 46, 2018

24. De Buck M, Gouwy M, Wang JM, Van Snick J, Opdenakker G, Struyf S and Van Damme J: Structure and expression of different serum amyloid A (SAA) variants and their concentration-dependent functions during host insults. Curr Med Chem 23: 1725-1755, 2016.

25. Sun L and Ye RD: Serum amyloid A1: Structure, function and gene polymorphism. Gene 583: 48-57, 2016.

26. De Buck M, Gouwy M, Wang JM, Van Snick J, Proost P, Struyf S and Van Damme J: The cytokine-serum amyloid A-chemokine network. Cytokine Growth Factor Rev 30: 55-69, 2016.

27. Jensen LE and Whitehead AS: Regulation of serum amyloid A protein expression during the acute-phase response. Biochem J 334: 489-503, 1998.

28. Heo TH, Wahler J and Suh N: Potential therapeutic implications of IL-6/IL-6R/gp130-targeting agents in breast cancer. Oncotarget 7: 15460-15473, 2016.

29. Schmidt-Arras D and Rose-John S: IL-6 pathway in the liver: From physiopathology to therapy. J Hepatol 64: 1403-1415, 2016.

30. Jiang Z and Zhu L: Update on molecular mechanisms of corticosteroid resistance in chronic obstructive pulmonary disease Pulm Pharmacol Ther 37: 1-8, 2016.

31. Liu X, Jones GW, Choy EH and Jones SA: The biology behind interleukin-6 targeted interventions. Curr Opin Rheumatol 28: 152-160, 2016.

32. Li W, Zhou Y and Zhang T: Expressions of serum TSLP, SAA, and CRP in COPD patients. J Chin Physician 20: 46-49, 2018.

33. Chen YWR, Leung JM and Sin DD: A systematic review of diagnostic biomarkers of COPD exacerbation. PLoS One 11: e0158843, 2016.

34. Landi A, Broadhurst D, Vernon SD, Tyrrell DL and Houghton M: Reductions in circulating levels of IL-16, IL-7 and VEGF-A in myalgic encephalomyelitis/chronic fatigue syndrome. Cytokine 78: 27-36, 2016.

35. Flores RJ: The use of circulating SAA and CXCL4 to predict outcome of osteosarcoma at diagnosis. Cancer Res 76: 459, 2016.

36. Fu G-Q, Xia J and Wang G: Clinical values of SAA and hs-CRP in the diagnosis of postoperative infection in patients with ovarian tumor. Zhongguo Jiceng Yiyao 24: 1069-1072, 2017 (In Chinese) 